\title{
Protection Group Effects During $\alpha, \gamma$-Diol Lignin Stabilization Promote High-Selectivity Monomer Production
}

\author{
Wu Lan, Masoud Talebi Amiri, Christopher M. Hunston, and Jeremy S. Luterbacher*
}

\begin{abstract}
Protection groups were introduced during biomass pretreatment to stabilize lignin's $\alpha, \gamma$-diol group during its extraction and prevent its condensation. Acetaldehyde and propionaldehyde stabilized the $\alpha, \gamma$-diol without any aromatic ring alkylation, which significantly increased final product selectivity. The subsequent hydrogenolysis catalyzed by $\mathrm{Pd} / \mathrm{C}$ generated lignin monomers at near-theoretical yields based on Klason lignin (48\% from birch, 20\% from spruce, $70 \%$ from high-syringyl transgenic poplar), and with high selectivity to a single 4-n-propanolsyringol product $(80 \%)$ in the case of the poplar. Unlike direct hydrogenation of native wood, hydrogenolysis of protected lignin with $\mathrm{Ni} / \mathrm{C}$ also led to high selectivity to this single product $(78 \%)$, paving the way to highselectivity lignin upgrading with base metal catalysts. The use of extracted lignin facilitated valorization of polysaccharides, leading to high yields of all three major biomass polymers to a single major product.
\end{abstract}

Co onversion of renewable resources, such as lignocellulosic biomass into chemicals, materials, and fuels, has attracted substantial attention in recent years because of limited fossil reserves and increasing environmental concerns over petroleum use. ${ }^{[1]}$ Lignin, a heteropolymer of phenylpropanoid units, is one of the three major components in lignocellulosic biomass and is the most abundant natural source of aromatic molecules. ${ }^{[2]}$ For this reason, lignin is often presented as a future feedstock for aromatic chemicals. ${ }^{[3]}$ However, few commercial processes or biorefinery schemes are being developed for lignin compared to other biopolymers such as cellulose or hemicellulose. These polysaccharides can be upgraded at high yields (ca. 70-100\%) to simple sugars, such as glucose and xylose, using relatively simple processes. ${ }^{[4]}$ In contrast, there are limited practical processes that can convert lignin at high yields and high selectivity to a narrow set of products, while remaining compatible with sugar production.

This production of sugars often involves biorefinery fractionation processes that use organic solvents ${ }^{[5]}$ or water $^{[6]}$ at high temperature with mineral acids to effectively remove lignin (and hemicellulose). But the resulting lignin systematically suffers from irreversible repolymerization that

[*] Dr. W. Lan, M. T. Amiri, C. M. Hunston, Prof. J. S. Luterbacher Laboratory of Sustainable and Catalytic Processing Institute of Chemical Sciences and Engineering École polytechnique fédérale de Lausanne, EPFL 1015 Lausanne (Switzerland) E-mail: jeremy.luterbacher@epfl.ch Supporting information and the ORCID identification number(s) for the author(s) of this article can be found under: https://doi.org/10.1002/anie.201710838. limits its ability to be upgraded at high yields. Several studies have developed so-called "lignin first" extraction methods, where native wood is treated in the presence of a heterogeneous metal catalyst that allows for the rapid hydrogenation of lignin intermediates before they condense. ${ }^{[7]}$ However, these methods introduce catalyst separation issues from unreacted wood and usually result in at least partial conversion of the hemicellulose fraction of biomass, which limits their integration into current biorefinery processes.

We recently proposed a method enabling efficient lignin extraction and depolymerization that was compatible with the traditional upgrading of biomass polysaccharides. ${ }^{[8]}$ Formaldehyde added during lignin extraction reacted with the $\alpha, \gamma$-diol on the side chain of lignin by forming a 1,3-dioxane structure. This protection group completely suppressed undesirable repolymerization, providing high monomer yield (47$78 \%$ ) in the subsequent hydrogenolysis with a precious metal catalyst. However, alkylation of aromatic rings by formaldehyde and unselective hydrogenolysis complicated the product mixture, leading to the production of six to 10 major monomers. Herein, we report for the first time that by modifying the protection group on $\alpha, \gamma$-diol-protected lignin, high yields and selectivities can be achieved for only two products (4- $n$-propanolguaiacol (POHG) and 4- $n$-propanolsyringol (POHS)). Furthermore, when a transgenic highsyringyl poplar is used, only a single major product (POHS) is obtained.

We investigated the effect of adding different diol protection reagents during the lignin extraction process on the final lignin monomer yield after hydrogenolysis (Figure 1; Supporting Information, Table S1). As a control, we performed the same extraction without a protection reagent (in triplicate) and the average yield was $14 \mathrm{wt} \%$ with a $95 \%$ confidence interval between 13-15 wt \%. Therefore, any experiment resulting in a yield value outside this range was very likely an effect of protection reagents. The first reagents that we tried were aldehydes that form cyclic acetal with the $\alpha, \gamma$-diol of the lignin side chain. All aldehydes, except for hydroxymethylfurfural, led to a statistically significant improvement in monomer yield. In the case of acetaldehyde and propionaldehyde, the monomer yields were 37 and $42 \mathrm{wt} \%$, respectively, which are comparable to that obtained with formaldehyde (46 wt \%). Interestingly, no alkylated aromatic monomers were detected, resulting in higher selectivity to ethylsyringol and ethylguaiacol. Ketal groups, especially acetonide, are some of the most common diol protection groups in carbohydrate chemistry. Therefore, acetone, butanone, and other related reagents that protect 1,2 and 1,3-diol, were also tested. In all of these experiments, some protective effect was detected during lignin extraction, 


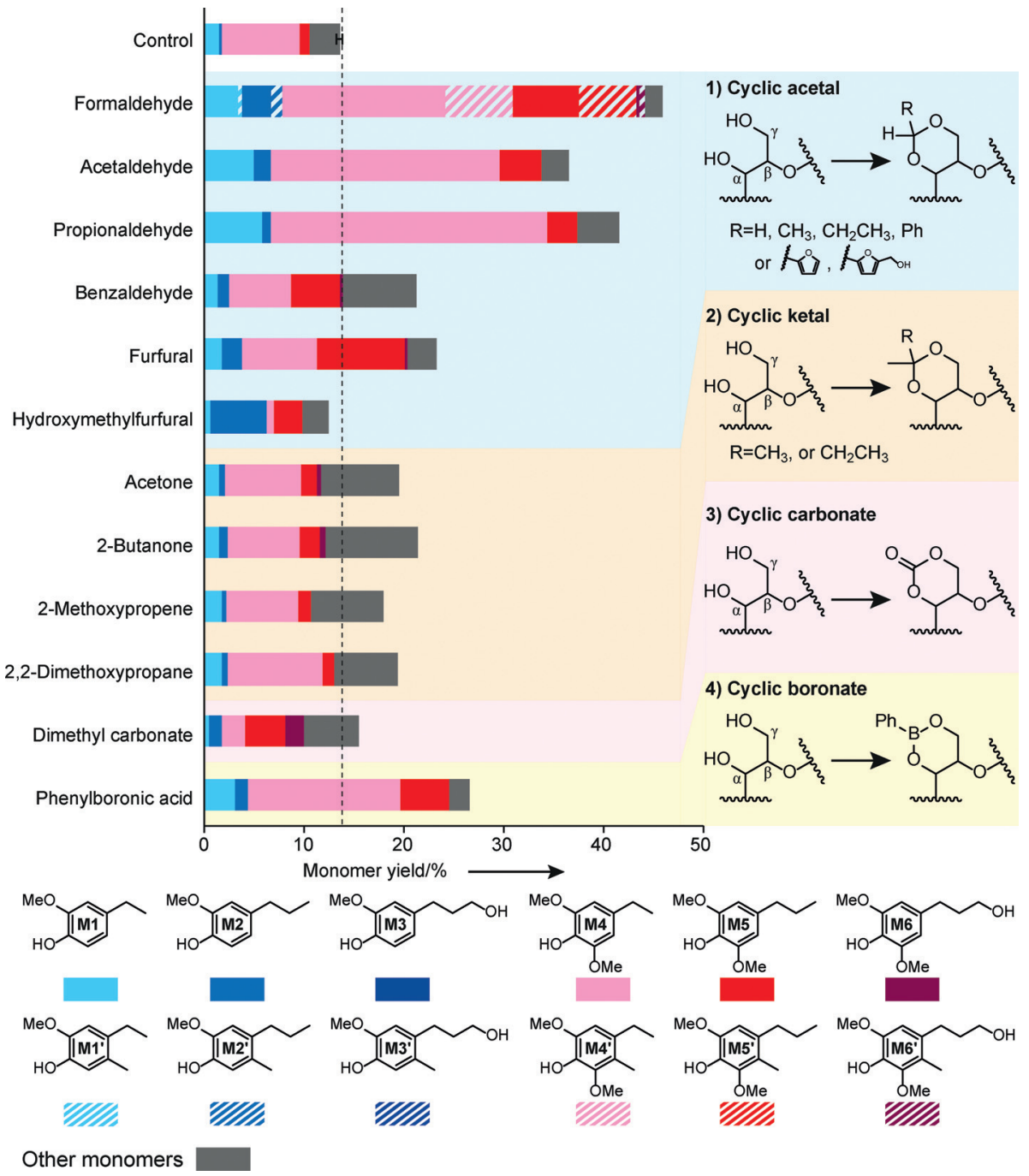

Figure 1. Hydrogenolysis of birch lignin extracted with the addition of different protection reagents in dioxane. Extraction condition: $80^{\circ} \mathrm{C}, 5 \mathrm{~h}$; hydrogenolysis condition: $\mathrm{Ru} / \mathrm{C}, 250^{\circ} \mathrm{C}, 40$ bar of $\mathrm{H}_{2}, 15 \mathrm{~h}$. Monomer yields are given based on Klason lignin content (Supporting Information, Table S1).

that of methylene-acetal-protected VG $(92 \%)$. The formation of the acetal-protected structures were confirmed by nuclear magnetic resonance (NMR; Figure 2A,B). According to the heteronuclear single quantum correlation (HSQC) spectra, the major compound of the mixture was the acetal-protected VG with trace amounts of unreacted VG. A detailed inspection of the HSQC and homonuclear correlation (COSY) spectra revealed no peaks originating from $-\mathrm{CH}_{2} \mathrm{OH}$ or $-\mathrm{CH}_{2} \mathrm{CH}_{2} \mathrm{OH}$ in the corresponding region, indicating that no hydroxyethylation or hydroxypropylation occurred during the reaction. After a subsequent hydrogenolysis reaction, no products with additional ethyl/propyl side chains on the aromatic ring were detected (Supporting Information, Figure S3), confirming the results obtained with native lignin in birch (Figure 1). Lignin extracted from birch in the presence of both acetand propionaldehyde were also prepared for NMR characterization (Figure 2C,D) and confirmed the structure observed with the model compound. Contours corresponding to each structure were integrated to calculate the syringyl/ guaiacyl $(\mathrm{S} / \mathrm{G})$ ratio and the percentage of different interlinkages. Results showed that more than $90 \%$ of the $\beta-O-4$ structure was

resulting in moderate but statistically significant monomer yield improvements after hydrogenolysis. We also tested carbonate and boronate protection groups that are known to react with alcohol groups under alkaline conditions. The use of dimethyl carbonate and phenylboronic acid as protection reagents resulted in monomer yields of 16 and $27 \mathrm{wt} \%$, respectively (above the yield of the control experiment; Supporting Information, Section 2.2). This study demonstrated that, while all diol protection groups were effective in stabilizing lignin to some degree, aldehydes were the most effective. Both acetaldehyde and propionaldehyde showed especially remarkable activity in protecting the $\alpha, \gamma$-diol position and preventing condensation during lignin extraction and pretreatment conditions.

To better understand the reaction mechanism, we performed reactions using the common lignin dimeric model compound veratrylglycerol- $\beta$-guaiacyl ether (VG) and acetor propionaldehyde. The yields of ethylidene-acetal- and propylidene-acetal-protected VG were $90 \%$ and $86 \%$, respectively, after a 30 minute reaction-slightly lower than protected by an ethylidene or propylidene acetal group, which was consistent with the model compound study.

The absence of aromatic ring alkylation, when acet- or propionaldehyde were used as protection reagents instead of formaldehyde, reduced the number of products by half, allowing for high selectivity lignin upgrading (Figure 3). We (and others) have previously shown that hybrid poplar in which the ferulate 5-hydroxylase $(\mathrm{F} 5 \mathrm{H})$ gene was overexpressed contained a much higher proportion of syringyl units in the lignin (more than $95 \%$ ) ${ }^{[9]}$ resulting in higher content of cleavable $\beta-O-4$ ethers. $^{[8]}$ We therefore used F5H poplar as a feedstock to get higher monomer yields and a higher product selectivity to syringyl monomers. The results of hydrogenolysis of lignin extracted with the addition of propionaldehyde are shown in Figure 4 (Supporting Information, Table S2). Using the same extraction conditions, a $54 \%$ monomer yield was obtained with propionaldehyde compared to $75 \%$ for formaldehyde. With formaldehyde, long extraction time benefited lignin extraction. However, considering that propylidene acetal is less stable than methylene 


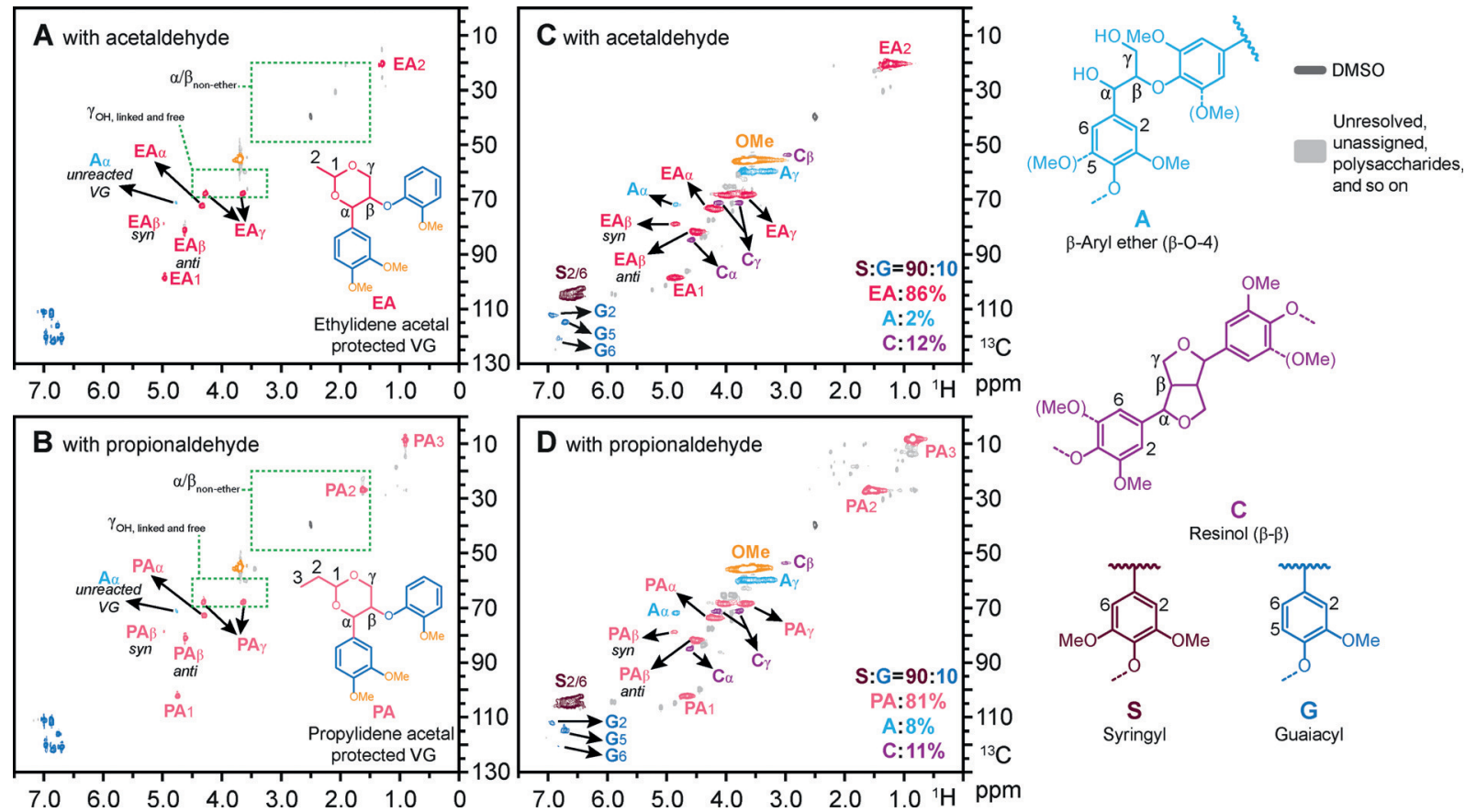

Figure 2. HSQC spectra of the product mixture resulting from the reaction between veratrylglycerol- $\beta$-guaiacyl ether and $A$ ) acetaldehyde, B) propionaldehyde, or isolated birch lignin extracted in the presence of C) acetaldehyde or D) propionaldehyde. The contour areas were integrated to calculate the S:G ratio and interunit linkage distributions. S:G equals $\left(\mathrm{S}_{2 / 6}\right) / 2:\left(\mathrm{G}_{2}\right) ; A A \alpha, A \alpha$, plus $C \alpha$ equals $100 \%$. $G$ reen dashed lines indicate the regions of hydroxypropyl and hydroxyethyl correlations. Key: dimethyl sulfoxide (DMSO), veratrylglycerol- $\beta$-guaiacyl ether (VG).

acetal, a longer extraction time could favor the partial cleavage of the protection groups and lead to some lignin repolymerization. Therefore, we reduced the lignin extraction time to $2.5 \mathrm{~h}$, which increased the monomer yield to $65 \mathrm{wt} \%$. Under these conditions, about $20 \%$ of guaiacyl monomers were obtained, which is higher than what would be expected based on the native lignin (ca. $5 \%$ ). This discrepancy was likely a result of the demethoxylation of syringyl units during hydrogenolysis over $\mathrm{Ru} / \mathrm{C}$. We also assumed that ethylphenols were mainly produced from propanolphenols after cleaving of the $-\mathrm{CH}_{2} \mathrm{OH}$ group over $\mathrm{Ru} / \mathrm{C}$ (Figure 3). Model compound studies using POHS and propylsyringol (PS) as reactants for hydrogenolysis demonstrated the cleavage of $-\mathrm{CH}_{2} \mathrm{OH}$ and demethoxylation under the same reaction conditions (Supporting Information, Figure S4). We lowered the temperature and/or time of hydrogenolysis to suppress this demethoxylation and $\mathrm{CH}_{2} \mathrm{OH}$ cleavage (Figure 4). When decreasing the reaction time from $15 \mathrm{~h}$ to $5 \mathrm{~h}$, a $73 \%$ monomer yield was obtained. Additionally, the POHS and POHG products were favored at a lower temperature: $60 \%$ selectivity to POHS was reached at $200^{\circ} \mathrm{C}$ for $15 \mathrm{~h}$ over $\mathrm{Ru} / \mathrm{C}$ and the fraction of syringyl products increased to $88 \%$.

To further increase the selectivity to $\mathrm{POHS} / \mathrm{POHG}, \mathrm{Pd} / \mathrm{C}$ was used as the hydrogenolysis catalyst because of its low alcohol hydrogenation activity. ${ }^{[10]}$ By running hydrogenolysis at $200^{\circ} \mathrm{C}$ for $20 \mathrm{~h}$, a yield of $70 \%$ monomers was obtained with $80 \%$ selectivity to a single POHS product (89\% to POHS + POHG; Figure 4), which is a potential precursor of adhesives and polymers. HSQC spectra 


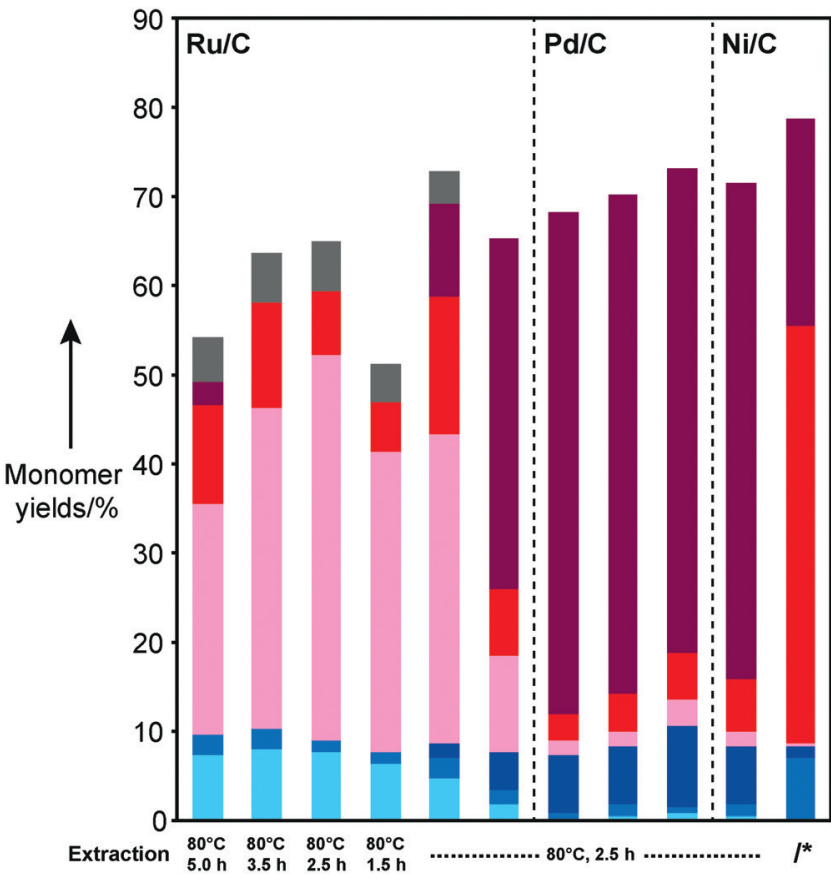

Extraction $5.0 \mathrm{~h} \quad 3.5 \mathrm{~h} \quad 2.5 \mathrm{~h} \quad 1.5 \mathrm{~h}$

Hydrogenolysis $250^{\circ} \mathrm{C}, 15 \mathrm{~h}$. $250^{\circ} \mathrm{C} 200^{\circ} \mathrm{C} 200^{\circ} \mathrm{C} 200^{\circ} \mathrm{C} 250^{\circ} \mathrm{C} 200^{\circ} \mathrm{C} 200^{\circ} \mathrm{C}$

Figure 4. Monomer yields (on the basis of Klason lignin) and product distribution resulting from the hydrogenolysis of propylidene-acetalprotected lignin extracted from $\mathrm{F} 5 \mathrm{H}$ poplar (Supporting Information, Table S2). Key: see Figure 1 for color-coding of molecules; *direct hydrogenolysis.

of the hydrogenolysis product mixtures further confirmed the monomers structure, and demonstrated full conversion of $\beta-O-4$ linkages of the lignin polymer (Supporting Information, Figure S5). This result is in agreement with previous work by Sels and colleagues, which also reported a high selectivity to POHG and POHS when treating birch sawdust mixed with $\mathrm{Pd} / \mathrm{C}$ in methanol to directly upgrade native lignin. ${ }^{[7 a]}$ Herein, we have achieved similar results with extracted lignin, as well as significantly higher selectivities to a single product (POHS) when using F5H-poplar. Nickel has often been proposed as a non-noble metal hydrogenolysis catalyst for lignin, producing mainly propylphenolic monomers during direct hydrogenolysis of untreated wood. When F5H-poplar was used during direct hydrogenolysis of untreated wood with $\mathrm{Ni} / \mathrm{C}$ in methanol, a relatively low product selectivity was obtained $(79 \%$ monomer yield with a $59 \%$ selectivity to PS and only $29 \%$ to POHS). Interestingly, when propionaldehyde-protected extracted lignin was used, a selectivity of $78 \%$ to POHS was achieved with an only slightly diminished yield of total monomers (72\%; Figure 4). The acetal protection group is likely causing a suppression of alcohol hydrogenation over nickel, but further study of the mechanism is still underway.

We also performed lignin extraction with acet- and propionaldehyde, as well as hydrogenolysis with $\mathrm{Pd} / \mathrm{C}$ with birch and spruce (Figure 5; Supporting Information, Figure S6). In all cases, more than $91 \%$ selectivity to POHS and POHG monomers was achieved. Total monomer yields were those that are typically expected for both wild-type species

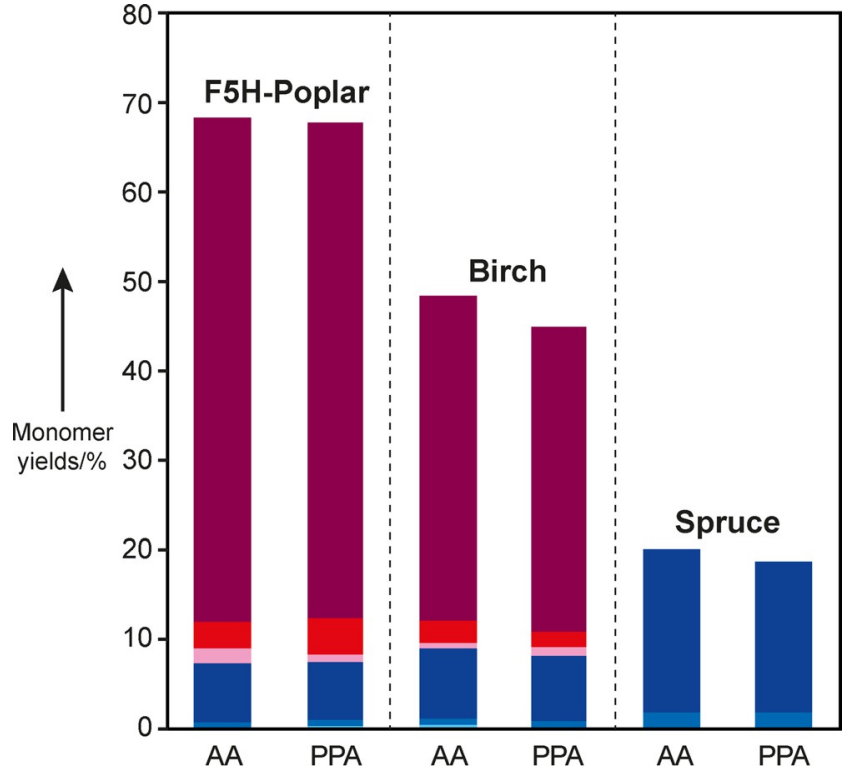

Figure 5. Monomer yields (on the basis of Klason lignin) and product resulting from the hydrogenolysis of lignin extracted from $\mathrm{F} 5 \mathrm{H}$ poplar, birch, and spruce with addition of acetaldehyde (AA) and propionaldehyde (PPA). The extraction conditions were $80^{\circ} \mathrm{C}, 2.5 \mathrm{~h}$ for $\mathrm{F} 5 \mathrm{H}$ poplar and birch, and $3.5 \mathrm{~h}$ for spruce. The hydrogenolysis was performed at $200^{\circ} \mathrm{C}$, with 40 bar of $\mathrm{H}_{2}$ for $15 \mathrm{~h}$ with $\mathrm{Pd} / \mathrm{C}$ (Supporting Information, Table S2). Key: see Figure 1 for color-coding of molecules; ferulate 5 -hydroxylase $(\mathrm{F} 5 \mathrm{H})$.

based on their fraction of interunit ether linkages: $48 \%$ and $20 \%$ for birch and spruce, respectively (extraction yields were within $10 \%$ of the yield from direct hydrogenolysis of untreated wood). ${ }^{[1]}$ The relatively low yield from spruce is consistent with the low $\beta$-aryl ether content in softwood lignin, as reported elsewhere. ${ }^{[11 \mathrm{a}, \mathrm{b}]}$ Nevertheless, because softwoods have only guaiacyl units, the selectivity to the single POHG product was $91 \%$.

Besides lignin depolymerization, recovery of carbohydrates was also evaluated. Unlike direct hydrogenolysis of native wood, which causes irreversible conversion of some of the carbohydrates, this method generated clean cellulose or acetal-protected carbohydrates (mostly xylose). The latter could be easily deprotected by aqueous acidic treatment ( $77 \%$ of xylose was obtained after $10 \mathrm{~min}$ at $120^{\circ} \mathrm{C}$ in $6 \mathrm{wt} \%$ $\mathrm{H}_{2} \mathrm{SO}_{4}$; Figure 6; Supporting Information, Figure S7 A). The leftover solids after pretreatment could undergo a typical enzymatic hydrolysis process without any further acid treatment, generating an $80 \%$ yield of glucose (Supporting Information, Figure S7B). The high enzymatic hydrolysis yield without any further acid treatment was a significant improvement over our previous work with formaldehyde, ${ }^{[8]}$ where the resulting cellulose required a harsh acid treatment to remove acetal groups that caused significantly reduced digestibilities (ca. 10\%). We suspect that any binding of propionaldehyde to cellulose was much more easily reversible than that of formaldehyde, and was likely removed even under the mild conditions $\left(50^{\circ} \mathrm{C}, \mathrm{pH} 5\right)$.

In summary, we developed a high-yield lignin extraction and depolymerization process that is compatible with most biorefinery schemes and can, depending on the biomass 


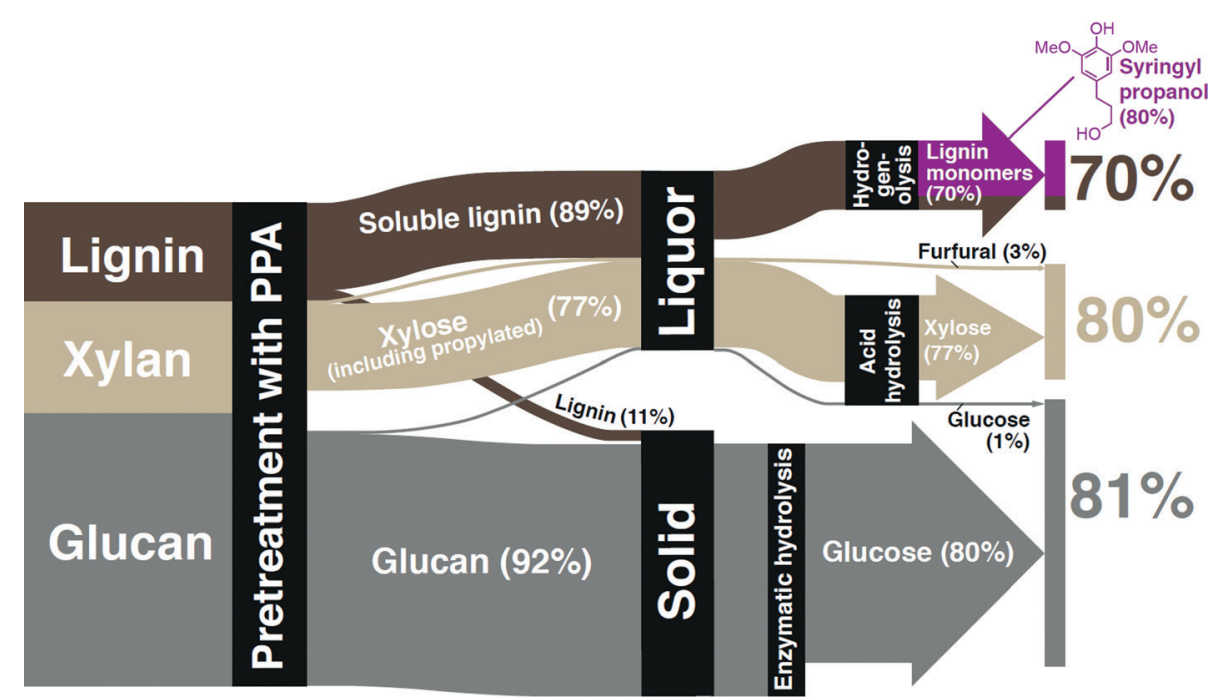

Figure 6. Mass balance of polysaccharides (glucan and xylan) and lignin fractions of biomass (Supporting Information, entry 14 in Table S3, and Figure S7).
[1] C. Z. Li, X. C. Zhao, A. Q. Wang, G. W. Huber, T. Zhang, Chem. Rev. 2015, 115, 11559-11624.

[2] W. Boerjan, J. Ralph, M. Baucher, Annu. Rev. Plant Biol. 2003, 54, $519-546$.

[3] R. Rinaldi, R. Jastrzebski, M. T. Clough, J. Ralph, M. Kennema, P. C. A. Bruijnincx, B. M. Weckhuysen, Angew. Chem. Int. Ed. 2016, 55, 8164-8215; Angew. Chem. 2016, 128, 8296-8354.

[4] J. S. Luterbacher, D. M. Alonso, J. A. Dumesic, Green Chem. 2014, $16,4816-4838$.

[5] a) W. Lan, C. F. Liu, R. C. Sun, J. Agric. Food Chem. 2011, 59, 8691 $8701 ;$ b) J. S. Luterbacher, J. M. Rand, D. M. Alonso, J. Han, J. T. Youngquist, C. T. Maravelias, B. F. Pfleger, J. A. Dumesic, Science 2014, 343, 277-280; c) X. J. Pan, N. Gilkes, J. Kadla, K. Pye, S. Saka, D. Gregg, K. Ehara, D. Xie, D. Lam, J. Saddler, Biotechnol. Bioeng. 2006, 94, 851-861.

source, produce a single product at high selectivity. To our knowledge, we are reporting the highest selectivities obtained for a single product from hardwood lignin depolymerization, and the instance of a non-precious metal being used for highselectivity lignin hydrogenolysis with molecular hydrogen. Because lignin is extracted under typical pretreatment conditions, both xylose and glucose can be produced at high yields as well, leading to mostly single products from all three major biopolymers.

\section{Acknowledgements}

This work was supported by the Swiss Competence Center for Energy Research: Biomass for a Swiss Energy Future through the Swiss Commission for Technology and Innovation grant KTI.2014.0116. We thank J. Ralph, C. Chapple, and R. Meilan for providing $\mathrm{F} 5 \mathrm{H}$ transgenic poplar and M. Studer for providing spruce and birch wood.

\section{Conflict of interest}

The authors declare no conflict of interest.

Keywords: biomass - depolymerization .

heterogeneous catalysis · hydrogenolysis · lignin

How to cite: Angew. Chem. Int. Ed. 2018, 57, 1356-1360 Angew. Chem. 2018, 130, 1370-1374
[6] N. Mosier, R. Hendrickson, N. Ho, M. Sedlak, M. R. Ladisch, Bioresour. Technol. 2005, 96, 1986-1993.

[7] a) S. Van den Bosch, W. Schutyser, S. F. Koelewijn, T. Renders, C. M. Courtin, B. F. Sels, Chem. Commun. 2015, 51, $13158-$ 13161; b) P. Ferrini, R. Rinaldi, Angew. Chem. Int. Ed. 2014, 53, 8634-8639; Angew. Chem. 2014, 126, 8778-8783.

[8] L. Shuai, M. T. Amiri, Y. M. Questell-Santiago, F. Heroguel, Y. D. Li, H. Kim, R. Meilan, C. Chapple, J. Ralph, J. S. Luterbacher, Science 2016, 354, 329-333.

[9] J. J. Stewart, T. Akiyama, C. Chapple, J. Ralph, S. D. Mansfield, Plant Physiol. 2009, 150, 621-635.

[10] a) I. Furikado, T. Miyazawa, S. Koso, A. Shimao, K. Kunimori, K. Tomishige, Green Chem. 2007, 9, 582-588; b) M. A. Dasari, P. P. Kiatsimkul, W. R. Sutterlin, G. J. Suppes, Appl. Catal. A 2005, 281, 225-231.

[11] a) S. Van den Bosch, W. Schutyser, R. Vanholme, T. Driessen, S. F. Koelewijn, T. Renders, B. De Meester, W. J. J. Huijgen, W. Dehaen, C. M. Courtin, B. Lagrain, W. Boerjan, B. F. Sels, Energy Environ. Sci. 2015, 8, 1748-1763; b) T. Parsell, S. Yohe, J. Degenstein, T. Jarrell, I. Klein, E. Gencer, B. Hewetson, M. Hurt, J. I. Kim, H. Choudhari, B. Saha, R. Meilan, N. Mosier, F. Ribeiro, W. N. Delgass, C. Chapple, H. I. Kenttamaa, R. Agrawal, M. M. Abu-Omar, Green Chem. 2015, 17, 14921499; c) Q. Song, F. Wang, J. Y. Cai, Y. H. Wang, J. J. Zhang, W. Q. Yu, J. Xu, Energy Environ. Sci. 2013, 6, 994-1007; d) N. Yan, C. Zhao, P. J. Dyson, C. Wang, L. T. Liu, Y. Kou, ChemSusChem 2008, 1, 626-629; e) C. Z. Li, M. Y. Zheng, A. Q. Wang, T. Zhang, Energy Environ. Sci. 2012, 5, 6383-6390.

Manuscript received: October 21, 2017

Accepted manuscript online: December 6, 2017

Version of record online: January 10, 2018 